\section{The chicken or the egg?}

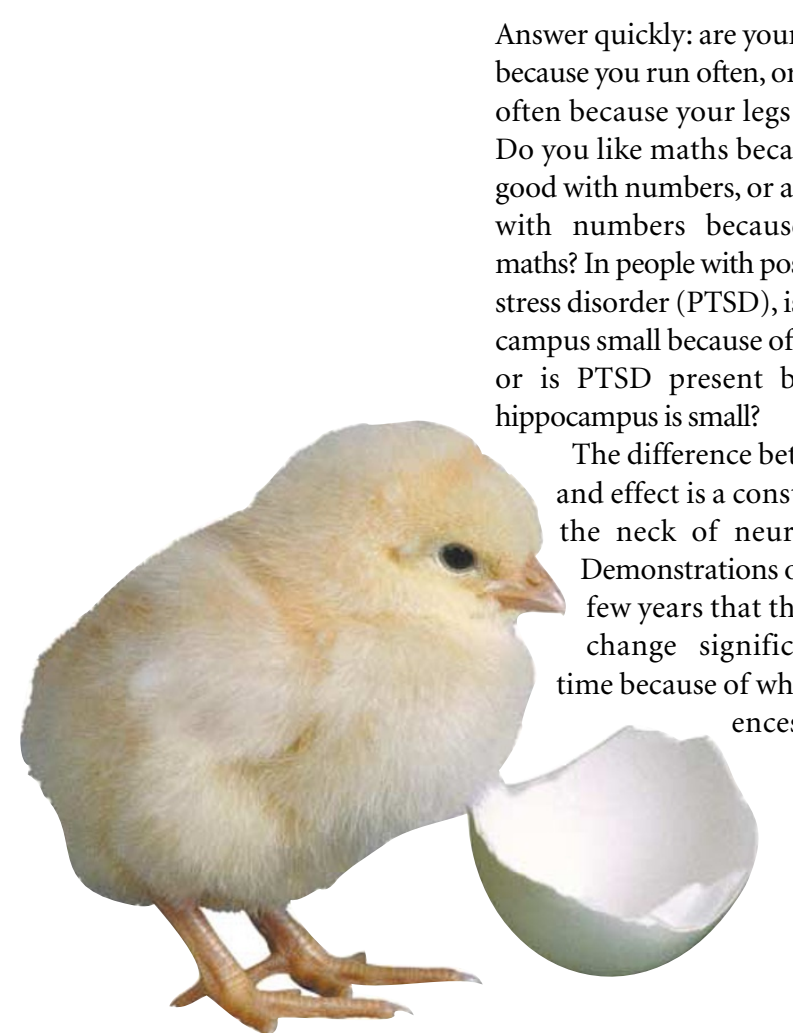

Answer quickly: are your legs strong often, or do you run yecause you are post-traumatic or is PTSD present because the The small?

The difference between cause of neuroscientists. thations over the past few years that the brain can change significantly over of what it experiences have only

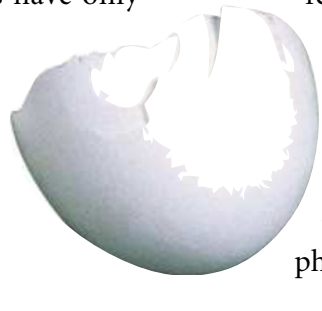

complicated matters. Braille readers have enlarged cortical representations of their fingertips; London taxi drivers, forced to know by heart their way around town, have an enlarged anterior hippocampus; women with chronic depression have a smaller hippocampus. But which comes first, the altered size of a brain area or the condition?

The usual way out is to look for a relationship between changes in the brain and the duration of practice or exposure. For example, the longer the time as a London cabbie, the larger the anterior part of the hippocampus; the longer the duration of chronic depression, the smaller the female hippocampus. Put these facts together with the discovery of continuous production of new neurons in the human hippocampus, and it seems perfectly plausible that this brain structure undergoes remodelling as it sees fit.

And this seemed to be the case with PTSD. In the 1990s, PTSD triggered by intense trauma and stress - two factors that cause neuronal atrophy and even death — was found to correlate with reduced hippocampal size in veterans of the Vietnam War. The severity of symptoms even correlated with the degree of hippocampal shrinkage relative to controls, which seemed to fit perfectly with the hypothesis of neuronal atrophy caused by stress.

Enter Mark Gilbertson and his data from 40 pairs of identical twins, published in the November issue of Nature Neuroscience. According to magnetic resonance imaging scans, the worse the symptoms of the disorder in combat veterans, the smaller the volume of the hippocampus in the twins who stayed at home.

Reduced hippocampal volume in the healthy twins correlated, of course, with a similar reduction in the veteran brother. The latter, however, was not related to combat severity - although those that developed PTSD had taken part in more severe combat than veterans who did not. The most parsimonious explanation is that, in the case of PTSD, reduced hippocampal size results neither from trauma nor from the disorder, but is instead a pre-existing condition that predisposes subjects to the development of PTSD once the brain is exposed to trauma. In this

\title{
DEVELOPMENT
}

\section{Signalling neuronal destiny}

During the development of the mammalian nervous system, progenitor cells seem to be driven by an intrinsic program to generate neurons and glia. But environmental cues also have an effect, as various growth factors and cytokines are involved in determining cell fate. For example, platelet-derived growth factor (PDGF) and neurotrophin-3 induce cortical progenitors to adopt a neuronal fate, whereas leukaemia inhibitory factor (LIF) and ciliary neurotrophic factor (CNTF) enhance glial differentiation. Until recently, little was known about the signalling mechanisms by which such factors mediate cortical neurogenesis and gliogenesis. So, LIF and CNTF have been shown to instruct glial differentiation by activating a receptor-associated tyrosine kinase, the Janus kinase ( JaK), which in turn induces various signal transduction pathways and activators of transcription to activate glial-specific genes. Now, Ménard et al. report on a new signalling mechanism that allows growth factors to induce progenitor cells to commit to a neuronal lineage.

CCAAT/enhancer-binding proteins

(CEBPs) belong to the leucine-zipper family of transcription factors and are known regulators of differentiation in liver, fat tissue and blood. Ménard et al. asked whether CEBPs might also influence cell differentiation during brain development. They isolated progenitor cells from the cortex of mouse embryos, cultured them with various growth factors, including PDGF, and tested whether knocking down CEBP activity in these cells disrupted cortical neurogenesis. Using adenoviral vectors to infect progenitor cells with an inhibitory form of CEBP, the authors found that CEBPs are critical for neuronal differentiation. Cells that were made deficient in CEBP activity rarely gave rise to neurons and were unable to transcribe neuron-specific genes. Instead, blocking CEBP activity in cortical progenitor cells caused them to remain in an undifferentiated state. Importantly, adding CNTF to the cultures of progenitor cells shifted the phenotypic profile; most CEBP-deficient progenitors became glia in response to added CNTF. Ménard et al. used a similar approach and site-directed mutagenesis at a CEBP phosphorylation site to illustrate the importance of the mitogen-activated protein kinase kinase (MEK) for activating CEBPs. So, it seems that growth factors such as PDGF can instruct cortical progenitor cells along a neuronal lineage by activating a MEK, which in turn induces CEBPs to activate neuron-specific programs of gene expression and inhibit gliogenesis. Owing to this dual effect, the activation of the MEK-CEBP pathway might be a mechanism whereby growth factors can bias progenitors to become neurons during cortical development.

Robin J. Lichtenwalner, Wake Forest University School of Medicine

(9) References and links ORIGINAL RESEARCH PAPER Ménard, C. et al. An essential role for a MEK-C/EBP pathway during growth-factorregulated cortical neurogenesis. Neuron 36, 597-610 (2002) FURTHER READING Bonni, A. et al. Regulation of gliogenesis in the central nervous system by the JAK-STAT gliogenesis in the central nervous system by the JAK
signaling pathway. Science $\mathbf{2 7 8}$, 477-483 (1997) 